\title{
Low-order distributed feedback optofluidic dye laser with reduced threshold
}

\author{
Wuzhou Song, ${ }^{1, a)}$ Andreas E. Vasdekis, ${ }^{1}$ Zhenyu $\mathrm{Li}^{2}$ and Demetri Psaltis ${ }^{1,2}$ \\ ${ }^{1}$ Optics Laboratory, IMT \& IBI, Swiss Federal Institute of Technology Lausanne (EPFL), \\ CH-1015 Lausanne, Switzerland \\ ${ }^{2}$ Electrical Engineering, California Institute of Technology, 1200 East California Boulevard, \\ Pasadena, California 91125, USA
}

(Received 19 November 2008; accepted 17 January 2009; published online 6 February 2009)

\begin{abstract}
We report the demonstration of low order distributed feedback (DFB) optofluidic dye lasers with reduced threshold. The laser chips were realized in polydimethylsiloxane using replica molding with two masters. A comparison between first, second, and third order DFB dye lasers was performed, while the second order DFB dye laser exhibited the lowest pump threshold of $78 \mathrm{~nJ} / \mathrm{pulse}$. Compared to previous reports on higher order Bragg grating structures, the pump threshold in this work is approximately 30-fold lower than the state of the art due to the reduction in the cavity losses and the more efficient pumping configuration. (C) 2009 American Institute of Physics.
\end{abstract}

[DOI: 10.1063/1.3079799]

In recent years, there has been rapid progress in developing optofluidic technology which has enabled a broad spectrum of novel optical toolboxes for integrated optics and lab-on-a-chip applications. ${ }^{1-3}$ To this end, optically pumped microfluidic dye lasers are of particular interest, since they exhibit the advantages of laser emission, combined with cost-effective processing and a wide choice of emission wavelengths.

Several resonator configurations have been demonstrated for microfluidic dye lasers such as the Fabry-Perot cavity, ${ }_{7}$ microdroplet, ${ }^{5}$ capillary tube, ${ }^{6}$ and photonic crystal fiber, ${ }^{7}$ while the distributed feedback (DFB) resonators were the more efficient ones which enable narrow linewidth single mode lasing operation and easy fabrication. ${ }^{8,9}$ A DFB microfluidic dye laser was first demonstrated by Balslev and Kristensen ${ }^{8}$ who used a 130th Brag grating in a multimode waveguide with a threshold of $20 \mu \mathrm{J} /$ pulse. Then a pure single mode optofluidic dye laser with significant lower pump threshold of $3.2 \mu \mathrm{J} /$ pulse was achieved by Li et al. ${ }^{9}$ However, microfluidic dye lasers still rely on bulky pumping laser systems due to their high thresholds, thus placing a restriction on their practicality. Hence, further reduction in the lasing threshold is highly desirable especially for compact diode pumping, as recently demonstrated for solid state organic semiconductor lasers.

In this letter, we present low order liquid core DFB microfluidic dye lasers with reduced lasing threshold. Our strategy to lower the pump threshold was based on reducing the out of plane diffraction losses by employing low order diffraction gratings and increasing the pumping efficiency by a longitudinal pumping geometry. The threshold gain $g_{\text {th }}$ for a DFB laser can be expressed as

$$
g_{\text {th }}=a+a_{m},
$$

where $a$ is the propagation loss coefficient in the cavity due to optical scattering, absorption, and out of plane diffraction and $a_{m}$ is the loss coefficient due to the finite reflection from the grating, also referred to as mirror loss coefficient. ${ }^{11} \mathrm{By}$

${ }^{a)}$ Electronic mail: wuzhou.song@epfl.ch. decreasing the grating order, both propagation loss $a$ and mirror loss $a_{m}$ can be decreased due to the reduced out of plane diffraction and enhanced in-plane coupling, respectively. By overcoming the previous technical limitation, we developed a two-step replica molding process that allowed fabricating low order DFB structures with the periods down to $200 \mathrm{~nm}$. In addition, the relationship between the gain at threshold $g_{\text {th }}$ and the pump power at threshold $P_{\text {th }}$ can be determined by

$$
g_{\text {th }}=\frac{2 \kappa \eta_{a} p_{\text {th }}}{V h v},
$$

where $\kappa$ is the product of the confinement factor, singlet exciton lifetime, photoluminescence quantum yield, and stimulated emission cross section, $\eta_{a}$ is the absorption efficiency of the pump light, $V$ is the cavity volume, and $h v$ is the pump photon energy.

As shown in Fig. 1(a), the laser chip was entirely made of polydimethylsiloxane (PDMS). It consisted of two monolithic layers: the top one had a microfluidic channel acting as the waveguide structure [Fig. 1(b)] while the bottom one had an array of nanogrooves acting as the DFB grating [Fig. 1(c)]. After aligning and bonding them together [Fig. 1(a)], the microfluidic channel was filled with a

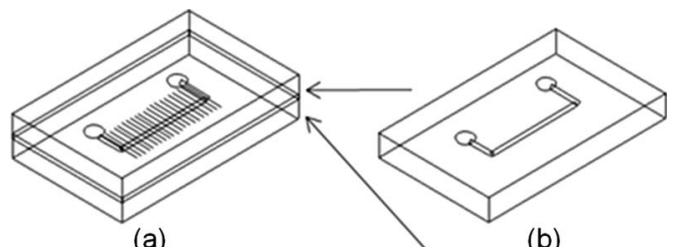

(a)

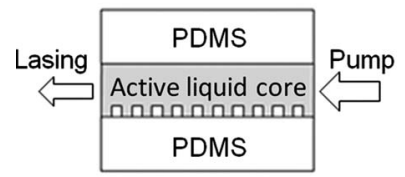

(d)

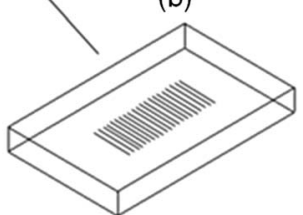

(c)
FIG. 1. [(a)-(c)] The fabrication steps followed to construct the DFB dye laser. (d) Schematic cross section of an optofluidic DFB dye laser. 


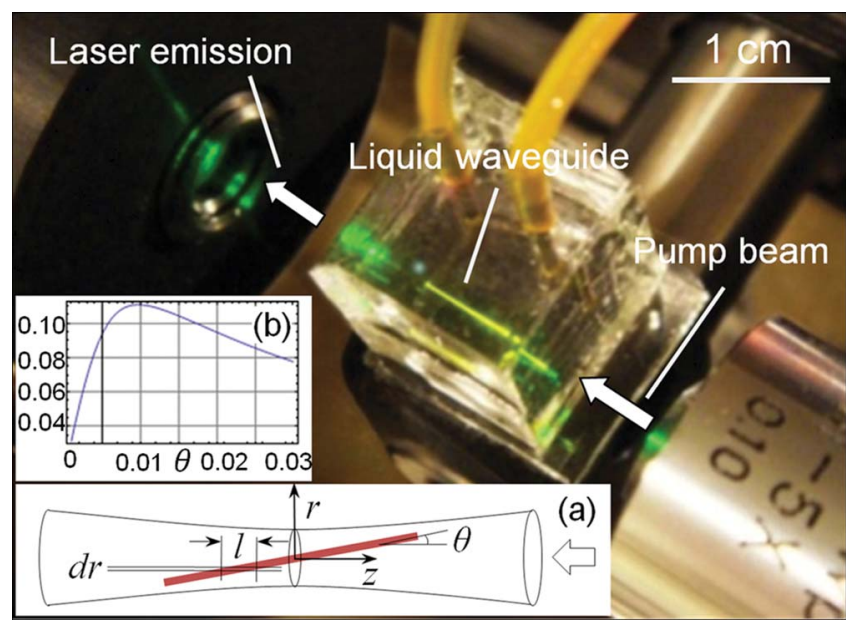

FIG. 2. (Color online) The picture of experimental setup for longitudinal pumping. Insets (a) illustrate the longitudinal pumping geometry and (b) the calculated dependence of the absorption efficiency as a function of the angle.

rhodamine $6 \mathrm{G}$ solution $(1 \mathrm{mg} / \mathrm{ml})$ with a slightly higher refractive index than that of PDMS $\left(n_{\mathrm{PDMS}}=1.4123\right)$. The dye solvent had a refractive index of 1.4140 produced by mixing water and dimethylsulfoxide. The waveguide had crosssectional dimension of $4 \times 4 \mu \mathrm{m}^{2}$. All the gratings were 5 $\mathrm{mm}$ long while the first, second, and third order DFB gratings had periods of 204, 408, and $612 \mathrm{~nm}$, respectively.

The top and bottom PDMS layers were prepared by standard replica molding method, respectively [Figs. 1(b) and $1(c)]$. The master mold for microfluidic channel was defined using standard UV lithography on a $4 \mu \mathrm{m}$ thick SU-8 film on a silicon wafer. The master grating mold was defined by electron beam lithography. The electron-beam resist was a $100 \mathrm{~nm}$ thick hydrogen silsesquioxane (HSQ) film coated on a silicon wafer. Each line had an approximate width of $100 \mathrm{~nm}$. The patterns of the structured SU-8 and HSQ films were transferred into two PDMS slabs (Dow Corning Sylgard 184) by replica molding, then the two PDMS slabs were immediately aligned and bonded.

As shown in Fig. 2 of the experimental setup, a longitudinal pumping method was implemented to improve the pumping efficiency. The laser beam from the $Q$-switched $\mathrm{Nd}$ :yttrium aluminum garnet laser (wavelength of $532 \mathrm{~nm}$, pulse duration of $4.5 \mathrm{~ns}$ ) was focused through a $5 \times$ magnification objective lens, thus forming a low divergence beam near the focus point (beam waist radius of $20 \mu \mathrm{m}$, divergence of $50 \mathrm{mrad}$ ). As shown in the inset of Fig. 2(a), to get maximum pump efficiency, the waveguide needs to be at a small tilt angle $\theta$ relative to the pump beam axis. The absorbed power $P$ can be expressed as

$$
P=\int_{-a / 2-L \sin \theta}^{a / 2+L \sin \theta} I(z, r)\left(1-e^{-\beta l}\right) a d r,
$$

where $I(z, r)$ is the intensity distribution of Gaussian beam and $z=r / \tan \theta, L$ is the waveguide length, $a$ denotes both the waveguide width and thickness, $l$ is the effective absorption length $(l=a / \sin \theta)$, and $\beta$ is the absorption coefficient. Therefore the absorption efficiency $\eta_{a}$ can be written as $\eta_{a}=P / P_{0} ; P_{0}$ is the total power of the input Gaussian beam. As shown in the inset graph of Fig. 2(b), the simulation result gives an optimized $\eta=11 \%$ when $\theta=0.01$ rad. Com-

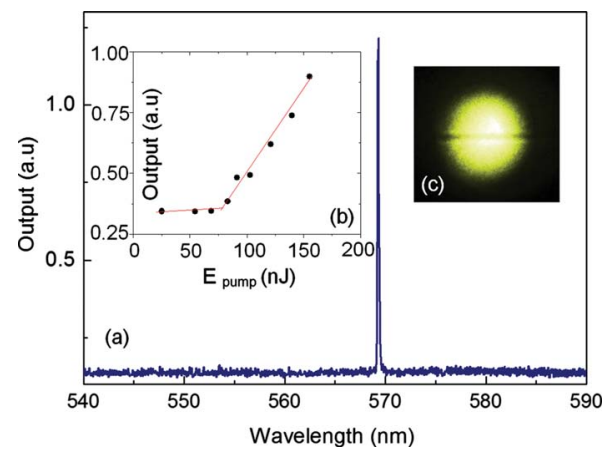

FIG. 3. (Color online) (a) The typical laser emission spectrum above the threshold from second order DFB optofluidic dye laser. (b) The plot of output energy vs the pumping energy. (c) The intensity pattern of the edge emitting laser beam from second order DFB dye laser.

pared to the absorption efficiency of $1.6 \%$ for transverse pumping (with cylindrical lens of $f=5 \mathrm{~mm}$ ), an enhancement factor of 7 in the absorption efficiency can be realized in the longitudinal pumping scheme.

A typical single mode lasing spectrum above threshold from the second order DFB dye laser is shown in Fig. 3(a). The laser mode appears at the wavelength of $569.1 \mathrm{~nm}$. The wavelength is $\sim 1.5 \%$ lower than calculations due to the PDMS shrinkage after solidification. The laser had a linewidth of less than $0.2 \mathrm{~nm}$. Figure 3(b) shows the input-output relationship of this laser. The threshold pump energy was measured to be $78 \mathrm{~nJ} /$ pulse. As shown in Fig. 3(c), the edge emitting laser beam pattern was also characterized by a charge-coupled device camera. The divergence of the laser beam was measured to be about $12^{\circ}$. The good quality of the lasing beam is attributed to the symmetric cross section of liquid waveguide cavity. According to the output energy from one end, the energy efficiency of the second order dye laser is calculated to be $2 \%$ at $300 \mathrm{~nJ} /$ pulse pump energy.

To investigate the effect of the grating order on the threshold pump energy, the first, second, and third order diffraction gratings were tested. The results are plotted in Fig. 4. In contrast to the expected behavior, the second order DFB microfluidic dye laser was found to exhibit the lowest average pump threshold of $90 \mathrm{~nJ} /$ pulse, lower even than the first order of $133 \mathrm{~nJ} / \mathrm{pulse}$. This is attributed to the fabrication limitation of replica molding with soft PDMS. Due to its low modulus and high viscosity, the commercial PDMS used for this experiment is unsuitable for replication of patterns below $250 \mathrm{~nm} .{ }^{12}$ Hence the grating depth of the first order on PDMS is lower than that of second order, thus resulting in weaker coupling in the first order. The third order DFB laser

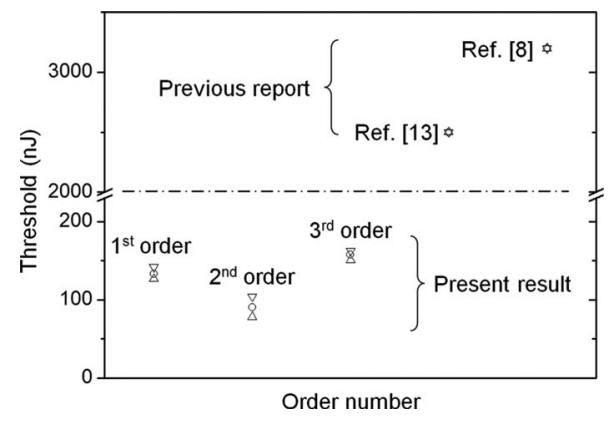

FIG. 4. The threshold pump energy comparison between different order DFB dye lasers. 
has much higher average pump threshold of $157 \mathrm{~nJ} /$ pulse than the second one. This is mainly attributed to the lower coupling coefficients and higher out of plane diffraction losses of the third order grating than the second order one at the same conditions of grating depth and waveguide configuration. Except for the previous microfluidic dye lasers which have much larger spectral linewidth, ${ }^{6,13}$ we compare the pump threshold of optofluidic dye lasers which have single mode and narrow spectral linewidth up to date. The third order DFB dye laser reported by Gersborg-Hansen and Kristensen ${ }^{14}$ held the record threshold of $2.5 \mu \mathrm{J} /$ pulse in the past, and the 15 th order liquid core $\mathrm{DFB}$ dye laser by $\mathrm{Li}$ et $a{ }^{9}{ }^{9}$ had a threshold of $3.2 \mu \mathrm{J} /$ pulse. In comparison to those results, the second order DFB microfluidic dye laser presented in this work exhibits approximately 30-fold lower pump threshold energy. Beside the reduction in the out of plane diffraction loss due to the lower order, the more efficient pumping scheme is another main factor for reducing the pump threshold.

In future work, we will explore the implementation of high quality first order gratings in alternative materials. In addition, to realize a light-emitting diode pumped microfluidic dye laser, a further reduction in threshold is necessary and, consequently, a different microcavity design with even higher $Q$ factor and pumping light absorption efficiency should be taken into consideration.

${ }^{1}$ D. Psaltis, S. R. Quake, and C. Yang, Nature (London) 442, 381 (2006).

${ }^{2}$ C. Monat, P. Domachuk, and B. J. Eggleton, Nat. Photonics 1, 106 (2007).

${ }^{3}$ W. Z. Song, A. Q. Liu, S. Swaminathan, C. S. Lim, P. H. Yap, and T. C. Ayi, Appl. Phys. Lett. 91, 223902 (2007).

${ }^{4}$ B. Helbo, A. Kistensen, and A. Menon, J. Micromech. Microeng. 13, 307 (2003).

${ }^{5}$ S. X. Qian, J. B. Snow, H. M. Tzeng, and R. K. Chang, Science 231, 486 (1986).

${ }^{6}$ S. Lacey, I. M. White, Y. Sun, S. I. Shopova, J. M. Cupps, P. Zhang, X. F. Fan, and P. Zhang, Opt. Express 15, 15523 (2007).

${ }^{7}$ A. E. Vasdekis, G. E. Town, G. A. Turnbull, and I. D. W. Samuel, Opt. Express 15, 3962 (2007).

${ }^{8}$ S. Balslev and A. Kristensen, Opt. Express 13, 344 (2005).

${ }^{9}$ Z. Li, Z. Zhang, T. Emery, A. Scherer, and D. Psaltis, Opt. Express 14, 696 (2006).

${ }^{10}$ I. D. W. Samuel and G. A. Turnbull, Chem. Rev. (Washington, D.C.) 107, 1272 (2007).

${ }^{11}$ J. Ohtsubo, Semiconductor Lasers, 2nd ed. (Springer, Berlin, 2008), p. 61

${ }^{12}$ H. Schmid and B. Michel, Macromolecules 33, 3042 (2000).

${ }^{13}$ C. Peroz, J. C. Galas, L. Le Gratiet, and Y. Chen, Appl. Phys. Lett. 89, 243109 (2006).

${ }^{14}$ M. Gersborg-Hansen and A. Kristensen, Appl. Phys. Lett. 89, 103508 (2007). 